\title{
A stochastic flow arising in the study of local times JON WARREN
}

\author{
Department of Statistics, University of Warwick, Coventry CV4 7AL, UK.
}

\begin{abstract}
A stochastic flow of homeomorphisms of $\mathbb{R}$ previously studied by Bass and Burdzy [2] and Hu and Warren 4 is shown to arise in the study of the local times of Brownian motion. This leads to a new proof of the Ray-Knight theorems for the flow via the classical Ray-Knight theorems for Brownian motion.
\end{abstract}

\section{Introduction}

Let $\beta_{1}$ and $\beta_{2}$ be fixed real constants. Suppose $B$ is a Brownian motion on the real line issuing from 0 . Associated with the equation

$$
X_{t}(x)=x+B_{t}+\beta_{1} \int_{0}^{t} d s \mathbf{1}\left(X_{s}(x) \leq 0\right)+\beta_{2} \int_{0}^{t} d s \mathbf{1}\left(X_{s}(x)>0\right),
$$

is a stochastic flow. There exists a random flow of homeomorphisms of the real line $\left(X_{t} ; t \geq 0\right)$ so that $t \mapsto X_{t}(x)$ satisfies the above equation for all $x \in \mathbb{R}$. For each $t$ the map $x \mapsto X_{t}(x)$ is increasing and differentiable. We denote the derivative by $D X_{t}(x)$. It has been shown by Bass and Burdzy, 2, and $\mathrm{Hu}$ and Warren, [4, that for certain stopping times $T$ the process $x \mapsto D X_{T}(x)$ is a diffusion process. Such a result was called a Ray-Knight theorem for the flow.

Let $\left(W_{t} ; t \geq 0\right)$ be a real-valued Brownian motion starting from $W_{0}=\xi>0$. Let $T_{0}$ be the stopping time

$$
T_{0}=\inf \left\{t \geq 0: W_{t}=0\right\} .
$$

Let $(l(t, x) ; t \geq 0, x \in \mathbb{R})$ be the family of bi-continuous local times of $W$. The celebrated RayKnight theorem states that the process $x \mapsto l\left(T_{0}, x\right)$ is a diffusion. The problem studied by Warren and Yor [7] (actually a slight variant of it) was that of obtaining a description of $\left(W_{t} ; 0 \leq t \leq T_{0}\right)$ after conditioning on the values of the entire family of random variables $\left(l\left(T_{0}, x\right) ; x \in \mathbb{R}\right)$. A related problem was treated by Aldous, [1. The method of [7] was to write $W$ as a random transformation of a process $\hat{W}$, christened the Burglar process, which is independent of $\left(l\left(T_{0}, x\right) ; x \in \mathbb{R}\right)$. It was shown, using a skew product linking squared Bessel and Jacobi diffusions, that Ray-Knight type theorems hold for $\hat{W}$ itself. The principal result of this paper is the following theorem which establishes a close connection between the this problem and a stochastic flow of the Bass-Burdzy type. Let $l(t, x)$ be the local times of a real-valued Brownian motion $W$ as above. Define

$$
\lambda(t, x)=l\left(T_{0}, x\right)-l(t, x), \quad \text { for } x \in \mathbb{R}, \quad t \in\left[0, T_{0}\right] .
$$

Let $M=\sup \left\{W_{t}: t \in\left[0, T_{0}\right]\right\}$ and $T_{M}=\inf \left\{t: W_{t}=M\right\}$. Now define a family of random changes of scale via:

$$
\phi_{t}(x)=\int_{x}^{W_{t}} \frac{d z}{\lambda(t, z)} \quad \text { for } x \in(0, M), \quad t \in\left[0, T_{M}\right) .
$$

Each map $\phi_{t}$ is a homeomorphism between $(0, M)$ and $\mathbb{R}$ and satisfies $\phi_{t}\left(W_{t}\right)=0$. Next define a random clock via:

$$
A_{t}=\int_{0}^{t} \frac{d s}{\lambda\left(s, W_{s}\right)^{2}} \quad \text { for } 0 \leq t<T_{M} .
$$

We will see below that $A_{T_{M}-}=\infty$, almost surely.

Theorem 1. The family of maps $\left(X_{u} ; 0 \leq u<\infty\right)$ defined via

$$
X_{A_{t}}(x)=\phi_{t} \circ \phi_{0}^{-1}(x) \quad \text { for } x \in \mathbb{R}, \quad 0 \leq t<T_{M},
$$

is distributed as the Bass-Burdzy flow associated with (11) with $\beta_{1}=0$ and $\beta_{2}=1$. Moreover it is independent of $\left(l\left(T_{0}, x\right) ; x \in \mathbb{R}\right)$. 


\section{From the Burglar to a flow}

In this section we prove Theorem 1 According to this result, the flow $\left(X_{u} ; 0 \leq u<\infty\right)$ constructed from the Brownian motion $W$ is independent of the local times $\left(l\left(T_{0}, x\right) ; x \in \mathbb{R}\right)$. Because of this, Theorem 1 is intimately related to the problem considered in [7] of describing the process $W$ conditional on knowing these local times. One standard approach for studying this type of problem is the method of enlargement of filtrations. We assume that the Brownian motion $\left(W_{t} ; t \geq 0\right)$ is carried by a filtered probability space $\left(\Omega,\left(\mathcal{F}_{t}\right)_{t \geq 0}, \mathbb{P}\right)$. Let $\mathcal{L}=\sigma\left(l\left(T_{0}, x\right) ; x \in \mathbb{R}\right)$ and introduce the enlarged filtration $\left(\mathcal{F}_{t}^{*} ; t \geq 0\right)$ where $\mathcal{F}_{t}^{*}=\mathcal{F}_{t} \vee \mathcal{L}$. Typically we would seek to show that a martingale in the original filtration is a semimartingale with respect to the enlarged filtration, and to obtain some expression for its canonical decomposition as such. However in this case there is no reason to believe that $W$ is a semimartingale with respect to $\mathcal{F}_{t}^{*}$; Jacod's absolute continuity criteria, [5], does not hold. Thus another approach is required to generate examples of martingales and semi-martingales relative to $\mathcal{F}_{t}^{*}$.

For $x \in \mathbb{R}$ we denote by $T_{x}$ the first time $W$ reaches the level $x$. We adopt the usual convention that $\exp (-\infty)=0$.

Proposition 2. Let $\kappa>2$ be a constant, and let $a, \xi$ and $c$ be constants satisfying $W_{0}=\xi$ and $0<a<\xi<c$. The process

$$
M_{t}^{*}=\exp \left\{\frac{-\kappa^{2}}{2} \int_{a}^{W_{t}} \frac{d y}{\lambda(t, y)}-\frac{\kappa(\kappa-2)}{2} \int_{W_{t}}^{c} \frac{d y}{\lambda(t, y)}\right\} \quad t \leq T_{a} \wedge T_{c},
$$

$M_{t}^{*}=M_{T_{a} \wedge T_{c}}^{*}$ for $t>T_{a} \wedge T_{c}$, is an $\mathcal{F}_{t}^{*}$-martingale.

Admitting this proposition for the moment, let us see how it leads to a proof the main result. First note:

Lemma 1.

$$
\int_{a}^{c} \frac{d z}{\lambda(t, z)}-\int_{a}^{c} \frac{d z}{\lambda(0, z)}=\int_{0}^{t} \mathbf{1}\left(a \leq W_{s} \leq c\right) d A_{s}
$$

almost surely on the event $\left\{c<M, t<T_{M}\right\}$.

Proof. Notice that on the event $\left\{c<M, t<T_{M}\right\}$ we have, almost surely, $\lambda(s, z)$ being bounded away from 0 for all $z \in[a, c]$ and $s \in[0, t]$. Then the result is a consequence of applying the occupation time formula, see Exercise (1.15), Chapter VI of Revuz and Yor, [6], to obtain:

$$
\int_{a}^{c} d z \int_{0}^{t} \frac{d_{s} l(s, z)}{\lambda(s, z)^{2}}=\int_{0}^{t} \mathbf{1}\left(a \leq W_{s} \leq c\right) \frac{d s}{\lambda\left(s, W_{s}\right)^{2}} .
$$

We can use this lemma to verify that $A_{T_{M}-}=\infty$ almost surely. For we see that on $\{c<M\}$,

$$
A_{T_{M}-} \geq \int_{a}^{c} \frac{d z}{\lambda\left(T_{M}, z\right)}-\int_{a}^{c} \frac{d z}{\lambda(0, z)}
$$

The distributions of $\lambda\left(T_{M}, \cdot\right)$ and $\lambda(0, \cdot)$ can be described using the classical Ray-Knight theorems for Brownian motion, as in Lemma [6 and then it follows from asymptotics of squared Bessel processes, to be found in for instance in [], that almost surely,

$$
\lim _{\epsilon \downarrow 0} \frac{1}{\log (1 / \epsilon)} \int_{a}^{M-\epsilon} \frac{d z}{\lambda\left(T_{M}, z\right)}=\infty \quad \text { and } \quad \lim _{\epsilon \downarrow 0} \frac{1}{\log (1 / \epsilon)} \int_{a}^{M-\epsilon} \frac{d z}{\lambda(0, z)}=1 / 2 .
$$

We define a process $\left(B_{t} ; t \geq 0\right)$ on the sample space $\Omega$ which carries $W$ via

$$
B_{A_{t}}=\int_{W_{0}}^{c} \frac{d z}{\lambda(0, z)}-\int_{W_{t}}^{c} \frac{d z}{\lambda(t, z)} \quad \text { on }\left\{t<T_{c}\right\} \cap\{c<M\} .
$$

Note that this equation defines $B$ consistently as $c$ varies, and that $B$ is defined for arbitrarily large times by letting $c \uparrow M$ so that $T_{c} \uparrow T_{M}$ and $A_{T_{c}} \uparrow \infty$. 
Let $X$ be defined as in Theorem 1 Suppose that $0<x<M$ and $t<T_{M}$. Then for $c \in(x, M)$ satisfying $t<T_{c}$,

$$
\begin{aligned}
X_{A_{t}}\left(\phi_{0}(x)\right)-\phi_{0}(x) & =\int_{x}^{W_{t}} \frac{d z}{\lambda(t, z)}-\int_{x}^{W_{0}} \frac{d z}{\lambda(0, z)} \\
& =\int_{x}^{c} \frac{d z}{\lambda(t, z)}-\int_{W_{t}}^{c} \frac{d z}{\lambda(t, z)}-\int_{x}^{c} \frac{d z}{\lambda(0, z)}+\int_{W_{0}}^{c} \frac{d z}{\lambda(0, z)} \\
& =B_{A_{t}}+\int_{x}^{c} \frac{d z}{\lambda(t, z)}-\int_{x}^{c} \frac{d z}{\lambda(0, z)} \\
& =B_{A_{t}}+\int_{0}^{t} \mathbf{1}\left(X_{A_{s}}\left(\phi_{0}(x)\right) \geq 0\right) d A_{s} .
\end{aligned}
$$

Here on the last line we have used Lemma 1 together with the fact that for $s<T_{c}$,

$$
\mathbf{1}\left(x \leq W_{s} \leq c\right)=\mathbf{1}\left(x \leq W_{s}\right)=\mathbf{1}\left(X_{A_{s}}\left(\phi_{0}(x)\right) \geq 0\right) .
$$

Time-changing equation (9), and putting $y=\phi_{0}(x)$, we see that $X_{t}(y)$ solves equation (10) driven by the process $B$.

We must next show that $B$ is a Brownian motion with respect to a suitable filtration. Using Lemma 1 we can re-write the martingale $M_{t}^{*}$ as,

$$
\frac{M_{t}^{*}}{M_{0}^{*}}=\exp \left\{-\kappa B_{A_{t}}-\frac{1}{2} \kappa^{2} A_{t}\right\} \quad \text { on }\left\{t<T_{a} \wedge T_{c}\right\} \cap\{c<M\} .
$$

By letting $a$ decrease to 0 and $c$ increase to $M$ we see that $\exp \left\{-\kappa B_{A_{t}}-\frac{1}{2} \kappa^{2} A_{t}\right\}$ is $\mathcal{F}_{t}^{*}$-local martingale on the stochastic interval $\left[0, T_{M}\right)$. Note here that $T_{M}$ is an $\mathcal{F}_{t}^{*}$-stopping time. Introduce that filtration $\left(\mathcal{G}_{u} ; u \geq 0\right)$ via

$$
\mathcal{G}_{u}=\mathcal{F}_{\alpha_{u}}^{*}, \quad u \in[0, \infty),
$$

where $\left(\alpha_{u} ; u \geq 0\right)$ is the inverse of $\left(A_{t} ; t \geq 0\right)$. The local martingale property is preserved by the time-change and we are able to conclude that $B$ is a Brownian motion by virtue of the next lemma.

Lemma 2. If $\left(B_{t} ; t \geq 0\right)$ is a continuous $\mathcal{G}_{t}$-adapted process such that

$$
\exp \left\{-\kappa B_{t}-\frac{1}{2} \kappa^{2} t\right\} \text { is a } \mathcal{G}_{t} \text {-local martingale }
$$

for at least two distinct and non-zero values of $\kappa$, then $\left(B_{t} ; t \geq 0\right)$ is a $\mathcal{G}_{t}$-Brownian motion.

Proof. By considering logarithms it is clear that $B_{t}$ is a $\mathcal{G}_{t}$ semimartingale. Let its canonical decomposition be $B_{t}=B_{0}+N_{t}+A_{t}$ where $A_{t}$ is a finite variation process and $N_{t}$ is a $\mathcal{G}_{t}$-local martingale. Applying Itô's formula to $Z_{t}^{(\kappa)}=\exp \left\{-\kappa B_{t}-\frac{1}{2} \kappa^{2} t\right\}$, we obtain

$$
Z_{t}^{(\kappa)}=Z_{0}^{(\kappa)}-\kappa \int_{0}^{t} Z_{s}^{(\kappa)} d B_{s}+\kappa^{2} / 2 \int_{0}^{t} Z_{s}^{(\kappa)}\left(d[N]_{s}-d s\right)
$$

Using this we see that the finite variation part of the local martingale $\frac{1}{\kappa} \int_{0}^{t} d Z_{s}^{(\kappa)} / Z_{s}^{(\kappa)}$, which must be identically zero, is given by

$$
-A_{t}+\frac{\kappa}{2}\left(d[N]_{s}-d s\right)
$$

From this we conclude that $A_{t}$ is identically zero, that is to say $B_{t}$ is a local martingale itself, and that $[B]_{t}=[N]_{t}=t$. Thus $B$ is a $\mathcal{G}_{t}$-Brownian motion by virtue of Itô's characterization.

Finally to complete the proof of Theorem 1 we must note that the Brownian motion $B$ is independent of the $\sigma$-algebra $\mathcal{G}_{0}$ which contains $\mathcal{L}$, and that since the stochastic differential equation (11) is exact by Zvonkin's observation, the flow $X$ is measurable with respect to $B$, and hence also independent of $\mathcal{L}$.

We turn now to proving Proposition 2 The principle tool used in constructing $\mathcal{F}_{t}^{*}$-martingales is a certain Markov property. Assume that the sample space $\Omega$ is the canonical space $\mathcal{C}([0, \infty), \mathbb{R})$ and $\left(W_{t} ; t \geq 0\right)$ realized as the co-ordinate process on $\Omega$. Then we can introduce the family $\left(\theta_{t} ; t \geq 0\right)$ of shift operators:

$$
W_{t} \circ \theta_{s}=W_{t+s}, \quad s, t \geq 0 .
$$


For $x \in \mathbb{R}$ let $\mathbb{P}^{x}$ be the probability measures on $\Omega$ under which the co-ordinate process $W$ is a Brownian motion starting from $x$. Then let $\mathbb{P}^{x, \ell}$ for $\ell \in L$ denote a version of the regular conditional probability for $\mathbb{P}^{x}$ given $l\left(T_{0}, \cdot\right)=\ell(\cdot)$, where $L$ is some suitable space of local time profiles.

Proposition 3. The process $\left(W_{t}, \lambda(t, \cdot) ; 0 \leq t<T_{0}\right)$ is Markovian relative to the filtration $\left(\mathcal{F}_{t}^{*} ; t \geq\right.$ $0)$ :

$$
\mathbb{E}^{x}\left[F \circ \theta_{t} \mid \mathcal{F}_{t}^{*}\right]=\mathbb{P}^{W_{t}, \lambda(t, \cdot)}(F), \quad \text { a.s. on }\left\{t<T_{0}\right\},
$$

for all $x>0$, and non-negative, measurable $F$ on $\Omega$.

Proof. Let $\nu$ be a finite measure supported in $(0, \infty)$. Observe that, on $\left\{t<T_{0}\right\}$,

$$
\mathcal{E}_{\nu}\left(\left(l\left(T_{0}, \cdot\right)\right)=\mathcal{E}_{\nu}\left((\lambda(t, \cdot)) \mathcal{E}_{\nu}(l(t, \cdot))\right.\right.
$$

where $\mathcal{E}_{\nu}(l)$ denotes

$$
\exp \left\{-\frac{1}{2} \int_{0}^{\infty} \nu(d y) l(y)\right\}
$$

If we define $F^{\nu}$ to be $F \mathcal{E}_{\nu}\left(l\left(T_{0}, \cdot\right)\right)$ then

$$
F^{\nu} \circ \theta_{t} \mathcal{E}_{\nu}(l(t, \cdot))=F \circ \theta_{t} \mathcal{E}_{\nu}\left(l\left(T_{0}, \cdot\right)\right) \quad \text { on }\left\{t<T_{0}\right\}
$$

Also put $G^{\nu}=\mathbb{P}^{W_{0}, l\left(T_{0}, \cdot\right)}(F) \mathcal{E}_{\nu}\left(l\left(T_{0}, \cdot\right)\right)$. Then $\mathbb{E}^{y}\left[G^{\nu}\right]=\mathbb{E}^{y}\left[F^{\nu}\right]$, for all $y>0$, and

$$
G^{\nu} \circ \theta_{t}=\mathbb{P}^{W_{t}, \lambda(t, \cdot)}(F) \mathcal{E}_{\nu}(\lambda(t, \cdot)) \quad \text { on }\left\{t<T_{0}\right\}
$$

Using these various observations and two applications of the Markov property of Brownian motion we have, for each $A \in \mathcal{F}_{t}$

$$
\begin{aligned}
\mathbb{E}^{x}\left[F \circ \theta_{t} \mathbf{1}_{A} \mathbf{1}_{\left(t<T_{0}\right)} \mathcal{E}_{\nu}\left(l\left(T_{0}, \cdot\right)\right)\right] & =\mathbb{E}^{x}\left[F^{\nu} \circ \theta_{t} \mathbf{1}_{A} \mathbf{1}_{\left(t<T_{0}\right)} \mathcal{E}_{\nu}(l(t, \cdot))\right] \\
& =\mathbb{E}^{x}\left[\mathbb{E}^{W_{t}}\left[F^{\nu}\right] \mathbf{1}_{A} \mathbf{1}_{\left(t<T_{0}\right)} \mathcal{E}_{\nu}(l(t, \cdot))\right] \\
& =\mathbb{E}^{x}\left[\mathbb{E}^{W_{t}}\left[G^{\nu}\right] \mathbf{1}_{A} \mathbf{1}_{\left(t<T_{0}\right)} \mathcal{E}_{\nu}(l(t, \cdot))\right] \\
& =\mathbb{E}^{x}\left[G^{\nu} \circ \theta_{t} \mathbf{1}_{A} \mathbf{1}_{\left(t<T_{0}\right)} \mathcal{E}_{\nu}(l(t, \cdot))\right] \\
& =\mathbb{E}^{x}\left[\mathbb{P}^{W_{t}, \lambda(t, \cdot)}(F) \mathbf{1}_{A} \mathbf{1}_{\left(t<T_{0}\right)} \mathcal{E}_{\nu}\left(l\left(T_{0}, \cdot\right)\right)\right] .
\end{aligned}
$$

Since, the random variables $\mathbf{1}_{A}$ and $\mathcal{E}_{\nu}\left(l\left(T_{0}, \cdot\right)\right)$ generate $\mathcal{F}_{t}^{*}$ as $A$ and $\nu$ vary we are done.

Consider $F$ given by

$$
F=\mathbf{1}_{\left(T_{c}<T_{a}\right)} \exp \left\{\frac{-\kappa^{2}}{2} \int_{a}^{c} \frac{d y}{\lambda\left(T_{c}, y\right)}\right\}+\mathbf{1}_{\left(T_{a}<T_{c}\right)} \exp \left\{-\frac{\kappa(\kappa-2)}{2} \int_{a}^{c} \frac{d y}{\lambda\left(T_{a}, y\right)}\right\}
$$

where $0<a<\xi<c$, recalling $W_{0}=\xi$. Using the Markov property just proved, the martingale $M_{t}^{*}$ is constructed as

$$
\begin{aligned}
M_{t}^{*}=\mathbb{E}^{\xi}\left[F \mid \mathcal{F}_{t}^{*}\right] & =\mathbb{E}^{\xi}\left[F \circ \theta_{t} \mathbf{1}_{\left(t<T_{a} \wedge T_{c}\right)}+F \mathbf{1}_{\left(t>T_{a} \wedge T_{c}\right)} \mid \mathcal{F}_{t}^{*}\right] \\
& =\mathbb{P}^{W_{t}, \lambda(t, \cdot)}(F) \mathbf{1}_{\left(t<T_{a} \wedge T_{c}\right)}+F \mathbf{1}_{\left(t>T_{a} \wedge T_{c}\right)},
\end{aligned}
$$

and to complete the proof of Proposition 2 we will show that

$$
\mathbb{E}^{b}[F \mid \mathcal{L}]=G(b) \quad \text { a.s. }
$$

for all $b \in(a, c)$, where $G=G(b)$ is given by

$$
G=\exp \left\{\frac{-\kappa^{2}}{2} \int_{a}^{b} \frac{d y}{l\left(T_{0}, y\right)}-\frac{\kappa(\kappa-2)}{2} \int_{b}^{c} \frac{d y}{l\left(T_{0}, y\right)}\right\}
$$

As a first step in establishing (15), let us verify the integrated version $\mathbb{E}^{b}[F]=\mathbb{E}^{b}[G]$. According to the Ray-Knight theorems the local time process $\left(l\left(T_{0}, y\right) ; y \geq 0\right)$ is distributed, under $\mathbb{P}^{b}$, as a inhomogeneous diffusion: starting from zero it behaves as a squared Bessel process of dimension 2 for 
$y \in[0, b]$ and then behaves as a squared Bessel process of dimension 0 for $y \in[b, \infty)$. Conditioning on $l\left(T_{0}, a\right)=u, l\left(T_{0}, b\right)=v$ and $l\left(T_{0}, c\right)=w$, we have $\left(l\left(T_{0}, y\right) ; a \leq y \leq b\right)$ and $\left(l\left(T_{0}, y\right) ; b \leq y \leq c\right)$ are independent and distributed as squared Bessel bridges of dimension 2 and dimension 0 respectively. For any dimension $d \geq 0$ we will denote the density of the transition density of the $\delta$-dimensional squared Bessel process by $q_{t}^{\delta}(x, y)$, for $x, y, t>0$. Note that if $\delta=0$ then the transition kernel also has an atom at zero of size $q_{t}^{0}(x, 0)$. It is an consequence of the absolute continuity relations between the squared Bessel processes that:

$$
\mathbb{E}\left[\exp \left\{-\frac{\kappa^{2}}{2} \int_{0}^{t} d s / Z_{s}\right\} \mid Z_{0}=u, Z_{t}=v\right]=\left(\frac{u}{v}\right)^{\kappa / 2} \frac{q_{t}^{2 \kappa+2}(u, v)}{q_{t}^{2}(u, v)}
$$

if $Z$ is a squared Bessel with dimension 2; while

$$
\mathbb{E}\left[\exp \left\{-\frac{\kappa(\kappa-2)}{2} \int_{0}^{t} d s / Z_{s}\right\} \mid Z_{0}=v, Z_{t}=w\right]=\left(\frac{v}{w}\right)^{\kappa / 2} \frac{q_{t}^{2 \kappa}(v, w)}{q_{t}^{0}(v, w)}
$$

if $Z$ has dimension 0 . Combining these results gives

$$
\begin{array}{r}
\mathbb{E}^{b}\left[\exp \left\{-\frac{\kappa^{2}}{2} \int_{a}^{b} \frac{d y}{l\left(T_{0}, y\right)}-\frac{\kappa(\kappa-2)}{2} \int_{b}^{c} \frac{d y}{l\left(T_{0}, y\right)}\right\} \mid l\left(T_{0}, a\right)=u, l\left(T_{0}, b\right)=v, l\left(T_{0}, c\right)=w\right]= \\
\left(\frac{u}{w}\right)^{\kappa / 2} \frac{q_{b-a}^{2 \kappa+2}(u, v) q_{c-b}^{\kappa}(v, w)}{q_{b-a}^{2}(u, v) q_{c-b}^{0}(v, w)} .
\end{array}
$$

The joint law of $l\left(T_{0}, a\right), l\left(T_{0}, b\right)$ and $l\left(T_{0}, c\right)$ (restricted to $\left.w>0\right)$ is

$$
q_{a}^{2}(0, u) q_{b-a}^{2}(u, v) q_{c-b}^{0}(v, w) d u d v d w .
$$

So integrating out, noting that there is no contribution to the expectation from the event $\left\{l\left(T_{0}, c\right)=\right.$ $0\}$,

$$
\begin{aligned}
\mathbb{E}^{b}\left[\exp \left\{-\frac{\kappa^{2}}{2} \int_{a}^{b} \frac{d y}{l\left(T_{0}, y\right)}-\frac{\kappa(\kappa-2)}{2} \int_{b}^{c} \frac{d y}{l\left(T_{0}, y\right)}\right\}\right]= \\
\qquad \int_{0}^{\infty} d u \int_{0}^{\infty} d v \int_{0}^{\infty} d w\left(\frac{u}{w}\right)^{\kappa / 2} q_{a}^{2}(0, u) q_{b-a}^{2 \kappa+2}(u, v) q_{c-b}^{2 \kappa}(v, w) .
\end{aligned}
$$

Lemma 3. For any $u, w, s, t>0$, and any $\delta \geq 0$,

$$
\int_{0}^{\infty} d v q_{s}^{\delta+2}(u, v) q_{t}^{\delta}(v, w)=\frac{s}{s+t} q_{s+t}^{\delta+2}(u, w)+\frac{t}{s+t} q_{s+t}^{\delta}(u, w) .
$$

Proof. The identity can be rewritten as an identity-in-law. Let $Z_{t}^{\delta}(u)$ denote a random variable distributed as the value at time $t$ of a $\delta$-dimensional squared Bessel process starting from $u$. Then the claim is that

$$
Z_{t}^{\delta}\left(Z_{s}^{\delta+2}(u)\right) \stackrel{\text { law }}{=} 1_{(I=0)} Z_{s+t}^{\delta+2}(u)+1_{(I=1)} Z_{s+t}^{\delta}(u)
$$

where $I$ is a suitable Bernoulli random variable independent of other variables. Assume this holds for $\delta=0$, then several applications of the additive property,

$$
Z_{t}^{\delta+\delta^{\prime}}(x+y) \stackrel{\operatorname{law}}{=} Z_{t}^{\delta}(x)+Z_{t}^{\delta^{\prime}}(y)
$$

give us

$$
Z_{t}^{\delta}\left(Z_{s}^{\delta+2}(u)\right) \stackrel{\operatorname{law}}{=} Z_{t}^{\delta}\left(Z_{s}^{2}(u)+Z_{s}^{\delta}(0)\right) \stackrel{\text { law }}{=} Z_{t}^{0}\left(Z_{s}^{2}(u)\right)+Z_{s+t}^{\delta}(0) \stackrel{\operatorname{law}}{=} 1_{(I=0)} Z_{s+t}^{\delta+2}(u)+1_{(I=1)} Z_{s+t}^{\delta}(u) .
$$

Finally the $\delta=0$ case is easily explained by combining the Ray-Knight theorems with hitting probabilities for Brownian motion. 
Applying this lemma to the expression at (18) gives

$$
\mathbb{E}^{b}[G]=\frac{b-a}{c-a} \mathbb{E}^{c}\left[G_{+}\right]+\frac{c-b}{c-a} \mathbb{E}^{a}\left[G_{-}\right],
$$

where

$$
\begin{aligned}
& G_{+}=\exp \left\{\frac{-\kappa^{2}}{2} \int_{a}^{c} \frac{d y}{l\left(T_{0}, y\right)}\right\}, \\
& G_{-}=\exp \left\{-\frac{\kappa(\kappa-2)}{2} \int_{a}^{c} \frac{d y}{l\left(T_{0}, y\right)}\right\} .
\end{aligned}
$$

On the other hand, applying the strong Markov property of Brownian motion at $T_{a} \wedge T_{c}$ and using $\mathbb{P}^{b}\left(T_{a}<T_{c}\right)=c-b / c-a$ we obtain

$$
\mathbb{E}^{b}[F]=\frac{b-a}{c-a} \mathbb{E}^{c}\left[G_{+}\right]+\frac{c-b}{c-a} \mathbb{E}^{a}\left[G_{-}\right] .
$$

Thus, comparing this with the preceding (19), we have obtained

$$
\mathbb{E}^{b}[F]=\mathbb{E}^{b}[G] \quad \text { for all } a \leq b \leq c .
$$

Next we introduce a family of transformations. Suppose that $h:[0, \infty) \mapsto[0, \infty)$ is increasing with strictly positive derivative, and satisfies $h(0)=0, h(\infty)=\infty$. If $W$ is path beginning at $W_{0}>0$ let $T_{h} W \equiv W^{h}$ denote the transformed path satisfying

$$
h\left(W_{u}^{h}\right)=W_{H_{u}}, \quad \text { for } H_{u}<T_{0},
$$

and $W_{u}^{h}=W_{H_{u}}$ for $H_{u} \geq T_{0}$, where $u \mapsto H_{u}$ is the inverse of the increasing process

$$
t \mapsto \int_{0}^{t \wedge T_{0}} \frac{d s}{\left(h^{\prime} \circ h^{-1}\left(W_{s}\right)\right)^{2}}+\left(t-T_{0}\right)^{+} .
$$

Let $\nu$ be a positive, finite measure supported on $(0, \infty)$. The Sturm-Liouville equation

$$
\phi^{\prime \prime}=\phi \nu
$$

admits a unique, strictly positive, decreasing solution ( see Revuz-Yor [], appendix) satisfying $\phi(0)=1$. We shall denote this solution by $\Phi_{\nu}$. It has the following probabilistic characterization:

$$
\Phi_{\nu}(x)=\mathbb{E}^{x}\left[\mathcal{E}_{\nu}\left(l\left(T_{0}, \cdot\right)\right)\right] \quad \text { for } x \geq 0 .
$$

Define the probability measure $\mathbb{P}^{b, \nu}$ via

$$
\mathbb{P}^{b, \nu}=\frac{1}{\Phi_{\nu}(b)} \mathcal{E}_{\nu}\left(l\left(T_{0}, \cdot\right)\right) \cdot \mathbb{P}^{b} .
$$

Lemma 4. Let $\nu$ be a positive, finite measure supported on $(0, \infty)$. Take $h$ to be given by

$$
h(y)=\int_{0}^{y} \frac{d x}{\Phi_{\nu}(x)^{2}} .
$$

Then if $W$ has law $\mathbb{P}^{h(b)}$, the transformed process $T_{h} W$ has law $\mathbb{P}^{b, \nu}$.

Proof. Under $\mathbb{P}^{b}$ the density

$$
\frac{1}{\Phi_{\nu}(b)} \exp \left\{-\frac{1}{2} \int_{0}^{\infty} \nu(d y) l\left(T_{0}, y\right)\right\}
$$

is the terminal value of the exponential martingale

$$
\begin{aligned}
& \frac{\Phi_{\nu}\left(W_{t \wedge T_{0}}\right)}{\Phi_{\nu}(b)} \exp \left\{-\frac{1}{2} \int_{0}^{\infty} \nu(d y) l\left(t \wedge T_{0}, y\right)\right\}= \\
& \exp \left\{\ln \Phi_{\nu}\left(W_{t \wedge T_{0}}\right)-\ln \Phi_{\nu}\left(W_{0}\right)-\frac{1}{2} \int_{0}^{\infty} \nu(d y) l\left(t \wedge T_{0}, y\right)\right\}= \\
& \quad \exp \left\{\int_{0}^{t \wedge T_{0}} \frac{\Phi_{\nu}^{\prime}\left(W_{s}\right)}{\Phi_{\nu}\left(W_{s}\right)} d W_{s}-\frac{1}{2} \int_{0}^{t \wedge T_{0}}\left(\frac{\Phi_{\nu}^{\prime}\left(W_{s}\right)}{\Phi_{\nu}\left(W_{s}\right)}\right)^{2} d s\right\} .
\end{aligned}
$$


Whence, by Girsanov's formula, under $\mathbb{P}^{b, \nu}$,

$$
W_{t}-\int_{0}^{t \wedge T_{0}} \frac{\Phi_{\nu}^{\prime}\left(W_{s}\right)}{\Phi_{\nu}\left(W_{s}\right)} d s
$$

is a martingale. Now we just need to notice that the scale function corresponding to this drift $b(\cdot)=\Phi_{\nu}^{\prime}(\cdot) / \Phi_{\nu}(\cdot)$ is proportional to

$$
\int_{0}^{y} d x \exp \left\{-\int_{0}^{x} 2 b(z) d z\right\}=\int_{0}^{y} \frac{d x}{\Phi_{\nu}(x)^{2}} .
$$

Standard scale/speed measure arguments complete the proof.

Lemma 5. If the occupation measure of the path $W$ admits local times $l(t, y)$ then the transformed path $T_{h} W$ admits as local times $l^{h}(\cdot, \cdot)$ given by

$$
l^{h}(t, y)=\frac{1}{h^{\prime}(y)} l\left(H_{t}, h(y)\right), \quad \text { for } 0 \leq H_{t} \leq T_{0} .
$$

In particular $T_{0}^{h}$, the time at which the path $W^{h}$ first reaches zero, satisfies $H_{T_{0}^{h}}=T_{0}$ and

$$
l^{h}\left(T_{0}^{h}, y\right)=\frac{1}{h^{\prime}(y)} l\left(T_{0}, h(y)\right) .
$$

Proof. For any test function $f$, and $t$ satisfying $H_{t} \leq T_{0}$,

$$
\begin{aligned}
\int_{0}^{t} f\left(W_{s}^{h}\right) d s=\int_{0}^{t} f \circ h^{-1}\left(W_{H_{s}}\right) d s & =\int_{0}^{H_{t}} \frac{f \circ h^{-1}\left(W_{s}\right)}{\left(h^{\prime} \circ h^{-1}\left(W_{s}\right)\right)^{2}} d s \\
& =\int_{0}^{\infty} \frac{f \circ h^{-1}(x)}{\left(h^{\prime} \circ h^{-1}(x)\right)^{2}} l\left(H_{t}, x\right) d x=\int_{0}^{\infty} \frac{f(y)}{h^{\prime}(y)} l\left(H_{t}, h(y)\right) d y .
\end{aligned}
$$

Treating $T_{h}$ as an almost everywhere defined application from $\Omega$ to $\Omega$, we have with obvious notation,

$$
\begin{gathered}
F^{h}=F \circ T_{h}= \\
\mathbf{1}\left(T_{c}^{h}<T_{a}^{h}\right) \exp \left\{\frac{-\kappa^{2}}{2} \int_{a}^{c} \frac{d y}{\lambda^{h}\left(T_{c}^{h}, y\right)}\right\}+\mathbf{1}\left(T_{a}^{h}<T_{c}^{h}\right) \exp \left\{-\frac{\kappa(\kappa-2)}{2} \int_{a}^{c} \frac{d y}{\lambda^{h}\left(T_{a}^{h}, y\right)}\right\}= \\
\mathbf{1}\left(\left(T_{h(c)}<T_{h(a)}\right) \exp \left\{\frac{-\kappa^{2}}{2} \int_{h(a)}^{h(c)} \frac{d y}{\lambda\left(T_{h(c)}, y\right)}\right\}+\mathbf{1}\left(T_{h(a)}<T_{h(c)}\right) \exp \left\{-\frac{\kappa(\kappa-2)}{2} \int_{h(a)}^{h(c)} \frac{d y}{\lambda\left(T_{h(a)}, y\right)}\right\} .\right.
\end{gathered}
$$

Similarly we have

$$
\begin{aligned}
G^{h}=G \circ T_{h}=\exp \left\{\frac{-\kappa^{2}}{2} \int_{a}^{b} \frac{d y}{l^{h}\left(T_{0}^{h}, y\right)}-\frac{\kappa(\kappa-2)}{2} \int_{b}^{c} \frac{d y}{l^{h}\left(T_{0}^{h}, y\right)}\right\} \\
=\exp \left\{\frac{-\kappa^{2}}{2} \int_{h(a)}^{h(b)} \frac{d y}{l\left(T_{0}, y\right)}-\frac{\kappa(\kappa-2)}{2} \int_{h(b)}^{h(c)} \frac{d y}{l\left(T_{0}, y\right)}\right\} .
\end{aligned}
$$

Since the arguments leading to (23) hold equally if we replace throughout $a$ by $h(a), b$ by $h(b)$ and $c$ by $h(c)$, we deduce that

$$
\mathbb{E}^{h(b)}\left[F^{h}\right]=\mathbb{E}^{h(b)}\left[G^{h}\right] .
$$

Now suppose that $h$ and $\nu$ are associated as in Lemma 4 Then we have, by virtue of (28),

$$
\frac{1}{\Phi(b)} \mathbb{E}^{b}\left[F \mathcal{E}_{\nu}\left(l\left(T_{0}, \cdot\right)\right)\right]=\mathbb{E}^{b, \nu}[F]=\mathbb{E}^{h(b)}\left[F^{h}\right]=\mathbb{E}^{h(b)}\left[G^{h}\right]=\mathbb{E}^{b, \nu}[G]=\frac{1}{\Phi(b)} \mathbb{E}^{b}\left[G \mathcal{E}_{\nu}\left(l\left(T_{0}, \cdot\right)\right)\right] .
$$

The measure $\nu$ being arbitrary this proves that $\mathbb{E}^{b}[F \mid \mathcal{L}]=G$ and the proof of Theorem $\square$ is complete. 


\section{Ray-Knight theorems}

Suppose the Bass-Burdzy flow $X$ is constructed from a Brownian motion $W$ as in Theorem 1 Then

$$
D X_{A_{t}}(\cdot)=\frac{\phi_{t}^{\prime} \circ \phi_{0}^{-1}(\cdot)}{\phi_{0}^{\prime} \circ \phi_{0}^{-1}(\cdot)}=\frac{\lambda\left(0, \phi_{0}^{-1}(\cdot)\right)}{\lambda\left(t, \phi_{0}^{-1}(\cdot)\right)} .
$$

This allows us to convert statements concerning the distribution of the derivative of the flow into statements concerning the local times of $W$ and vice-versa. In this section we illustrate this by giving a proof of one of the Ray-Knight theorems for the flow by means of verifying the corresponding statement regarding Brownian local times.

The following result is part of Theorem 1.1 of [4, together with equation (1.18) there. Notice that, for each $x \in \mathbb{R}$, the process $t \mapsto D X_{t}(x)$ is constant except when $t \mapsto X_{t}(x)$ visits zero. In the case $\beta_{2}=1$ and $\beta_{1}=0$ then $t \mapsto X_{t}(x)$ is transient in the sense $X_{t}(x) \rightarrow \infty$ as $t \rightarrow \infty$ and so $t \mapsto D X_{t}(x)$ is eventually constant. It is thus meaningful to consider the limit $D X_{\infty}(x)$.

Theorem 4. Suppose that $\left(X_{t} ; t \geq 0\right)$ is the Bass-Burdzy flow with $\beta_{1}=0$ and $\beta_{2}=1$. Then $1 / D X_{\infty}(0)$ is uniformly distributed on $[0,1]$. Conditionally on $\left\{1 / D X_{\infty}(0)=y\right\}$ the processes $\left(Y_{x}^{+} ; x \geq 0\right)$ and $\left(Y_{x}^{-} ; x \geq 0\right)$ given by

$$
Y_{x}^{+}=\frac{1}{D X_{\infty}(x)} \quad \text { and } \quad Y_{x}^{-}=\frac{1}{D X_{\infty}(-x)},
$$

are independent diffusions on $[0,1]$, starting from $y$, with infinitesimal generators

$$
2 y(1-y) \frac{d^{2}}{d y^{2}}+2(1-y) \frac{d}{d y} \quad \text { and } \quad 2 y(1-y) \frac{d^{2}}{d y^{2}}+(2(1-y)-2 y) \frac{d}{d y},
$$

respectively.

It it should be noted that versions of this result hold for other values of $\beta_{1}$ and $\beta_{2}$, but by a combination of scaling and Girsanov transformations they may be deduced from the special case stated here.

The diffusions appearing in the above description of $D X_{\infty}$ are sometimes called Jacobi diffusions. A Jacobi diffusion with dimensions $d_{1}$ and $d_{2}$ has generator

$$
2 y(1-y) \frac{d^{2}}{d y^{2}}+\left(d_{1}(1-y)-d_{2} y\right) \frac{d}{d y}
$$

The behaviour at the boundaries 0 and 1 is determined from the corresponding dimension, $d_{1}$ for the boundary at 0 and $d_{2}$ for the boundary at 1 , according to the following rule. If $d=0$ the boundary is absorbing, if $0<d<2$ then the boundary is instantaneously reflecting, while if $d \geq 2$ then the boundary point is an inaccessible entrance point.

Next we give a description of Brownian local times corresponding to Theorem 4 Recall that the Brownian motion $W$ starts from $W_{0}=\xi>0$, that $M=\sup \left\{W_{t} ; t \in\left[0, T_{0}\right]\right\}$, and $T_{M}$ is the almost surely unique time such that $W_{T_{M}}=M$. Now as was noted earlier $A_{T_{M}-}=\infty$. So if we take $t=T_{M}$ in equation (30) we obtain

$$
\begin{array}{ll}
Y^{-}\left(\int_{\xi}^{x} \frac{d z}{l\left(T_{0}, z\right)}\right)=\frac{l\left(T_{0}, x\right)-l\left(T_{M}, x\right)}{l\left(T_{0}, x\right)} & \text { for } \xi \leq x<M, \\
Y^{+}\left(\int_{x}^{\xi} \frac{d z}{l\left(T_{0}, z\right)}\right)=\frac{l\left(T_{0}, x\right)-l\left(T_{M}, x\right)}{l\left(T_{0}, x\right)} & \text { for } 0<x \leq \xi,
\end{array}
$$

where $Y^{+}$and $Y^{-}$are defined as in Theorem 4 Thus Theorem 4 is equivalent to the following proposition.

Proposition 5. Suppose that processes $Y^{-}$and $Y^{+}$are determined from the local times of a Brownian motion via equations (31) and (32). Then $Y_{0}^{-}=Y_{0}^{+}$is uniformly distributed on [0,1] and conditionally on $Y_{0}^{-}=Y_{0}^{+}=y$ the processes $Y^{-}$and $Y^{+}$are independent Jacobi diffusions with dimensions $d_{1}^{-}=d_{2}^{-}=2$ and $d_{1}^{+}=2, d_{2}^{+}=0$ respectively. 
The principal tool used in proving this proposition is the skew-product involving Jacobi processes and squared Bessel processes, see 7]. Suppose that $(Z(t) ; t \geq 0)$ and $\left(Z^{\prime}(t) ; t \geq 0\right)$ are independent squared Bessel processes with dimensions $d$ and $d^{\prime}$ respectively and that $d+d^{\prime}>0$. Let $Z^{(+)}(t)=$ $Z(t)+Z^{\prime}(t)$ and suppose $Z^{(+)}(0)>0$. Define $Y$ via,

$$
Y\left(\int_{0}^{t} d s / Z^{(+)}(s)\right)=\frac{Z(t)}{Z^{(+)}(t)} .
$$

Then $Y$ is a Jacobi process with dimensions $d$ and $d^{\prime}$ independent of $Z^{(+)}$which is a squared Bessel process of dimension $d+d^{\prime}$. In fact, as was remarked in [7], the skew product holds also in the case that $Z$ and $Z^{\prime}$ are replaced by $\tilde{Z}$ and $\tilde{Z}^{\prime}$ which are obtained from $Z$ and $Z^{\prime}$ by

$$
\tilde{Z}_{t}=\frac{1}{u^{\prime}(t)} Z_{u(t)} \quad \text { and } \quad \tilde{Z}_{t}^{\prime}=\frac{1}{u^{\prime}(t)} Z_{u(t)}^{\prime}
$$

where $u$ is a strictly increasing, continuously differentiable function satisfying $u(0)=0$. We will make use of this in the case $u(t)=t /(h-t)$, in which case $\tilde{Z}$ and $\tilde{Z}^{\prime}$ are independent squared Bessel bridges leading to 0 over the interval $[0, h]$.

We may now proceed with the proof of Proposition 5 We decompose the path $\left(W_{t} ; 0 \leq t \leq T_{0}\right)$ about its maximum: let

$$
\begin{array}{lr}
W_{t}^{(1)}=W_{t} & \text { for } 0 \leq t \leq T_{M} \\
W_{t}^{(2)}=W_{T_{0}-t} & \text { for } 0 \leq t \leq T_{0}-T_{M} .
\end{array}
$$

Then conditionally on $M=m \in(\xi, \infty)$ the path segments $\left(W_{t}^{(1)} ; 0 \leq t \leq T_{M}\right)$ and $\left(W_{t}^{(2)} ; 0 \leq t \leq\right.$ $\left.T_{0}-T_{M}\right)$ are independent Bessel processes of dimension three run until first hitting the level $m$, started from $\xi$ and 0 respectively. Using this decomposition we obtain the description of the local times of $W$ in the following lemma, and then Proposition 5 and hence Theorem 4 follow by virtue of the skew product.

We use the notation $\mathbb{Q}_{x, y}^{d, h}$ to denote the law of a squared Bessel bridge of dimension $d$ leading from $x$ to $y$ over the interval $[0, h]$.

Lemma 6. Conditionally on $M=m$, the local time processes $l\left(T_{M}, \cdot\right)$ and $l\left(T_{0}, \cdot\right)-l\left(T_{M}, \cdot\right)$ are distributed as follows.

$\left(l\left(T_{M}, x\right) ; 0 \leq x \leq m\right)$ and $\left(l\left(T_{0}, x\right)-l\left(T_{M}, x\right) ; 0 \leq x \leq m\right)$ are independent

$l\left(T_{M}, \xi\right)$ and $l\left(T_{0}, \xi\right)-l\left(T_{M}, \xi\right)$ each have the exponential distribution with mean $2 \xi(m-\xi) / m$.

Conditionally on $l\left(T_{M}, \xi\right)=z$ the processes $\left(l\left(T_{M}, \xi-x\right) ; 0 \leq x \leq \xi\right)$ and $\left(l\left(T_{M}, \xi+x\right) ; 0 \leq x \leq\right.$ $m-\xi)$ are independent and are distributed as $\mathbb{Q}_{z, 0}^{0, \xi}$ and $\mathbb{Q}_{z, 0}^{2, m-\xi}$ respectively.

Conditionally on $l\left(T_{0}, \xi\right)-l\left(T_{M}, \xi\right)=z$ the processes $\left(l\left(T_{0}, \xi-x\right)-l\left(T_{M}, \xi-x\right) ; 0 \leq x \leq \xi\right)$ and $\left(l\left(T_{0}, \xi+x\right)-l\left(T_{M}, \xi+x\right) ; 0 \leq x \leq m-\xi\right)$ are independent and are distributed as $\mathbb{Q}_{z, 0}^{2, \xi}$ and $\mathbb{Q}_{z, 0}^{2, m-\xi}$ respectively.

Proof. $\left(l\left(T_{M}, x\right) ; 0 \leq x \leq m\right)$ are the local times of the path segment $W^{(1)}$ while $\left(l\left(T_{0}, x\right)-\right.$ $\left.l\left(T_{M}, x\right) ; 0 \leq x \leq m\right)$ are the local times of $W^{(2)}$. Thus their independence follows from that of $W^{(1)}$ and $W^{(2)}$.

Next it is well known (see [3] for instance) that the local times of a Bessel three process starting from zero taken at its hitting time of a level $m$ are distributed as $\mathbb{Q}_{0,0}^{2, m}$. So the distribution of $l\left(T_{0}, \xi\right)-l\left(T_{M}, \xi\right)$ is obtained as that of this bridge at time $\xi$ :

$$
\frac{q_{\xi}^{2}(0, z) q_{m-\xi}^{2}(z, 0)}{q_{m}^{2}(0,0)}=\frac{m}{2 \xi(m-\xi)} \exp \left\{-\frac{z m}{2 \xi(m-\xi)}\right\}
$$

where $q_{t}^{d}(x, y)$ are the transition densities of a squared Bessel process of dimension $d$. Moreover, by standard Markovian properties of bridges, a process distributed as $\mathbb{Q}_{0,0}^{2, m}$ can be constructed by conditioning on its value at time $\xi$ being $z$, and placing "back to back" two independent bridges 
distributed as $\mathbb{Q}_{z, 0}^{2, m-\xi}$ and $\mathbb{Q}_{z, 0}^{2, \xi}$. This proves that the distribution of $\left(l\left(T_{0}, x\right)-l\left(T_{M}, x\right) ; 0 \leq x \leq m\right)$ is as asserted.

Turn now to the process $\left(l\left(T_{M}, m-x\right) ; 0 \leq x \leq m\right)$ which is distributed of the local times of a Bessel three process starting from $\xi$ and taken at its hitting time of a level $m$. This is obtained by conditioning the inhomogeneous diffusion which starts from 0, evolves a squared Bessel process of dimension two until time $m-\xi$, and then evolves as a squared Bessel process of dimension zero, to have been absorbed at zero by time $m$. To see this, just note that according to the Ray-Knight theorems, Brownian motion started from $\xi$ and taken at its hitting time of $m$ has local times distributed as the unconditioned diffusion, and that the conditioning corresponds exactly to conditioning the path of the Brownian motion not to have reached zero before hitting level $m$. Thus the distribution of $l\left(T_{M}, \xi\right)$ is given by

$$
\frac{m}{\xi} q_{m-\xi}^{2}(0, z) q_{\xi}^{0}(z, 0)=\frac{m}{2 \xi(m-\xi)} \exp \left\{-\frac{z m}{2 \xi(m-\xi)}\right\}
$$

Finally, once again, by standard Markovian arguments we observe that this conditioned, inhomogeneous diffusion, can be constructed by conditioning on its value at time $\xi$ being $z$ and placing "back to back" two independent bridges distributed as $\mathbb{Q}_{z, 0}^{0, \xi}$ and $\mathbb{Q}_{z, 0}^{2, m-\xi}$.

Acknowledgment: Proposition 2 is a reworking of unpublished joint work with Marc Yor. This paper was written during a visit to the Department of Statistics at the University of California, Berkeley, and I would like to thank Jim Pitman and the Department for their hospitality.

\section{References}

[1] D. Aldous, Brownian excursion conditioned on its local time. Elect. Comm. in Probab. 3, 79-90, (1998).

[2] Bass, R.F. and Burdzy, K., Stochastic bifurcation models, Annals of Probability, 27, 50-108, (1999).

[3] C. Donati-Matin and M. Yor, Some Brownian functionals and their laws. Annals of Probability, 25:3, 1011-1058. (1997).

[4] Hu, Y. and Warren, J., Ray-Knight theorems for a stochastic flow, Stochastic processes and its applications, 86, 287-305, (2000).

[5] Jacod, J., Grossissement initial, hypothèse (H'), et théormè de Girsanov. In Grossissements de filtrations: exemples et applications, Lecture notes in Mathematics, 1118. Springer, (1985).

[6] D.Revuz and M.Yor, Continuous martingales and Brownian motion, Springer, (1998).

[7] Warren, J. and Yor, M., The Brownian burglar: conditioning Brownian motion by its local time process, Seminaire de Probabilités 32, 328-342, Lecture notes in Mathematics, 1709, Springer, (1998). 